\section{Major axes of stimulus figures do not account for dot displacements}

\author{
ALAN A. HARTLEY \\ Scripps College, Claremont, California 91711
}

Wenderoth's (1978) comments on my paper (Hartley, 1978) contain a serious oversight that taints the interpretation offered. Briefly, I showed observers dots placed within a square or an incomplete square on a piece of paper. They were asked to reproduce the position of the dot on a second, blank piece of paper. Glossing over details, the finding was that the reproduced position was displaced from the veridical position toward the location of the surrounding lines. There was no displacement toward axes of bilateral symmetry, that is, major axes. In a task requiring that a rod be set to the gravitational vertical, Beh and Wenderoth (1972), Beh, Wenderoth, and Purcell (1971), and Wenderoth (1973) had found that settings were displaced toward real lines of a surrounding figure or toward its axes of symmetry, whichever was nearer the rod. This would compensate for an illusory displacement away from the axes, termed a direct or repulsion effect. I noted that the dot displacements I observed did not agree with the results of the rod-setting experiments. Wenderoth challenges this, asserting that the observed displacements of dot positions are in perfect agreement with the rod-setting results. Wenderoth's argument runs as follows: The observers position the dot closer to the real lines because its apparent position has been displaced away from the axis of symmetry. His argument is sufficient to explain the displacements of dots that were $4 \mathrm{~cm}$ from the nearest real line and $2 \mathrm{~cm}$ from the axis of symmetry. The argument founders, however, since extension of the rod-setting results would predict that dots placed $2 \mathrm{~cm}$ from the real line and $4 \mathrm{~cm}$ from the axis of symmetry would be displaced away from the real line and toward the axis of symmetry (a direct effect of the real line). Instead, the reproductions were toward the real line. Thus, the results for dot positioning do not, as I stated correctly, agree with those for rod-setting.

On the pretext of pointing out findings that I overlooked in interpreting my results, Wenderoth then presents an extended recapitulation of two articles that became available after the submission of my paper (Wenderoth, 1977; Wenderoth \& Beh, 1977). Wenderoth provides a convenient summary of these important works, including one that may not be familiar to readers in the United States (Wenderoth, 1977). Although the discussion of these works is pre- sented as a critique of my paper, it is encouraging to note that the conclusions Wenderoth draws from the research they report agree closely with those I offered. First, it is clear that the major-axis hypothesis-the notion that displacements can be described as a function of the relation between the test stimulus and the axes of symmetry of the inducing figureis inadequate as a descriptive model. The results from my three-sided square and Wenderoth and Beh's (1977) incomplete squares establish this. Thus, no explanatory version of the model need be developed. Second, explanatory models based on lateral inhibition between lines of the inducing figure (or perhaps on spatial frequency analysis) seem to be attractive alternatives. My hypothesis that loci of neural interactions may account for the nonveridical perceptions is quite similar to the lateral inhibition model offered by Wenderoth and Beh (1977) (also see O'Toole \& Wenderoth, 1977). Both are loose, informal models that suggest the outlines of an adequate theory.

Finally, Wenderoth argues that dot-position methods do not necessarily bear on any theory of orientation judgments. The theories in question are not theories of orientation judgments, but rather theories of the effects of stimulus figures on nearby perceptual space. There is no a priori reason to suppose that either judgments of dot position or judgments of orientation would not provide an adequate test of theoretical predictions. In fact, dot judgments may provide sensitive probes of the microstructure of illusions, as Wenderoth's recent research on the Poggendorf illusion demonstrates (Wenderoth, Beh, \& White, 1978; Wenderoth, White, \& Beh, 1978).

In summary, the results I reported previously cannot be explained away as being in perfect agreement with the major-axis hypothesis. Moreover, taken together with results reported by Wenderoth and Beh (1977), they provide converging evidence for the inadequacy of the hypothesis that certain nonveridical perceptions are related in any simple way to the axes of bilateral symmetry of surrounding figures.

\section{REFERENCES}

Beh, H. C., \& Wenderoth, P. M. The effect of frame shape on the angular function of the rod-and-frame illusion. Perception \& Psychophysics, 1972, 11, 35-37.

Beh, H. C., Wenderoth, P. M., \& Purcell, A. T. The angular function of the rod-and-frame illusion. Perception \& Psychophysics, 1971, 9, 353-355.

Harteey, A. A. The major-axis effect: Axes of bilateral symmetry or loci of neural interactions? Perception \& Psychophysics, $1978,23,537-541$. 
O'Toole, B., \& Wenderoth, P. The tilt illusion: Repulsion and attraction effects in the oblique meridian. Vision Research, 1977, 17, 367-374.

Wenderoth, P. M. The effects of tilted outline frames and intersecting line patterns on judgments of vertical. Perception \& Psychophysics, 1973, 14, 242-248.

WENDEROTH, P. An analysis of the rod-and-frame illusion and its variants. In R. H. Day \& G. V. Stanley (Eds.), Studies in perception. Perth: University of Western Australia, 1977.

Wenderoth, P. The major-axis effect: Some comments on Hartley's experiment and analysis. Perception \& Psychophysics, $1978,24,572-574$
Wenderoth, P., \& BEH, H. Component analysis of orientation illusions. Perception, 1977, 6, 57-75.

Wenderoth, P., Beh, H., \& White, D. Alignment errors to both ends of acute- and obtuse-angle arms. Perception \& Psychophysics, 1978, 23, 475-482.

Wende Roth, P., White, D., \& Beh, H. The effect of peripheral and central fixation on a Poggendorf-like vernier alignment task. Perception \& Psychophysics, 1978, 24, 377-386.

(Received and accepted for publication August 8, 1979.) 